\title{
Design of Experimental Equipment Basic on Ultra-Low-Power MCU MSP430F4619
}

\author{
Jian Huang \\ (XiJing University, Xi'an 710123,China) \\ 565200245@qq.com
}

Keywords: MSP430; Segment LCD; Electronic Competition

\begin{abstract}
In order to meet the requirement of experimental and electronic design contest, in fulling exploring all kind of MSP430 of TI company, design MSP430F4619 experimental board. It include MSP430 minimal system, power unit, key unit, segment LCD unit, 16 SMD led unit, temperature sensor unit, photosensitive and thermal unit, relay unit, buzzer unit, LCD interface, wireless module, USB transfer interface, I2C interface, Hall-sensor, infrared sensor, JTAG interface. This equipment can do basic I/O operate experiment, LED light experiment, key and display experiment, acquisition of temperature and humidity experiment, wireless communication experiment, I2C communication experiment and many kinds of sensor experiment. It can replace simple $51 \mathrm{MCU}$ board, fits for university students of computer science and electronic information major, can be used for training of electronic design contest.
\end{abstract}

\section{Introduction}

SCM is widely used in industrial control systems, data acquisition systems, automatic testing systems, intelligent instruments and interfaces and other broad areas. Therefore, it has become a basic skill for students of computer, electronic information, automatic control and so on. [1-5]. However, the 51 single-chip microcomputer is a bit machine, a small number of pins, the function is limited, there are some limitations in data acquisition. In recent years, MSP430 company TI series microcontroller is widely used in the field of industrial control, intelligent instruments, household appliances etc. with its ultra low power consumption and excellent performance in power consumption, performance much more than $51 \mathrm{MCU}$. Therefore, in order to adapt to the requirements of teaching and electronic competition, so that students master the MSP430 high performance 16 bit microcontroller is very important.

To master SCM technology, practice is essential, it is necessary to develop a MSP430 microcontroller development board, making the students easy to use, can do hands-on programming experiments. At present, most of the domestic MSP430 microcontroller is based on the 1 series of MSP430, MSP430F169 or MSP430F149. is the MSP430 series of low-end products, low operating frequency, the clock is only $8 \mathrm{MHz}$, there are $48 \mathrm{IO}$ pins. Through the research of MSP430 device manual carefully and consider the basic teaching and competition requirements, put forward to make a MSP430F4619 MCU experimental device, the chip has $80 \mathrm{I} / \mathrm{O}$ pins, the clock frequency is $16 \mathrm{MHz}$, built-in 160 Segment LCD driver code ${ }^{[1-3]}$, very suitable for instrument display, 12 channel ADC12 and 2 DA12. Contains I2C, SPI and serial, very powerful. Very suitable for computer, telecommunications, automatic control and other professional students learning and practice.

\section{Hardware Composition}

The system is mainly controlled by MSP430F4619. Including MSP430 minimum system, power supply unit, a key unit, a liquid crystal display unit, 16 chip LED lamp circuit, temperature acquisition unit, photosensitive thermal interface, temperature and humidity sensor interface, relay, buzzer unit, LCD interface, wireless module, USB interface, I2C interface, Holzer sensor, infrared sensor, JTAG interface module. The following details describe some of the major modules. 


\section{MSP430F4619 Minimum System}

In the drawing schematic and printed board using Altium Designer13 software, the schematic diagram of the use of the network table to mark all the pins, drawings clear, each module is independent of each other [4-7]. The complete MSP430F4619 minimum system schematic is shown in figure 1. Including MSP430F4619 microcontroller, reset circuit, crystal oscillator circuit, JTAG interface circuit. Crystal oscillator which can choose $32.768 \mathrm{KHz}$ and $12 \mathrm{MHz}$ two. Each pin is identified by a network table and the same network identifier is connected to each other.

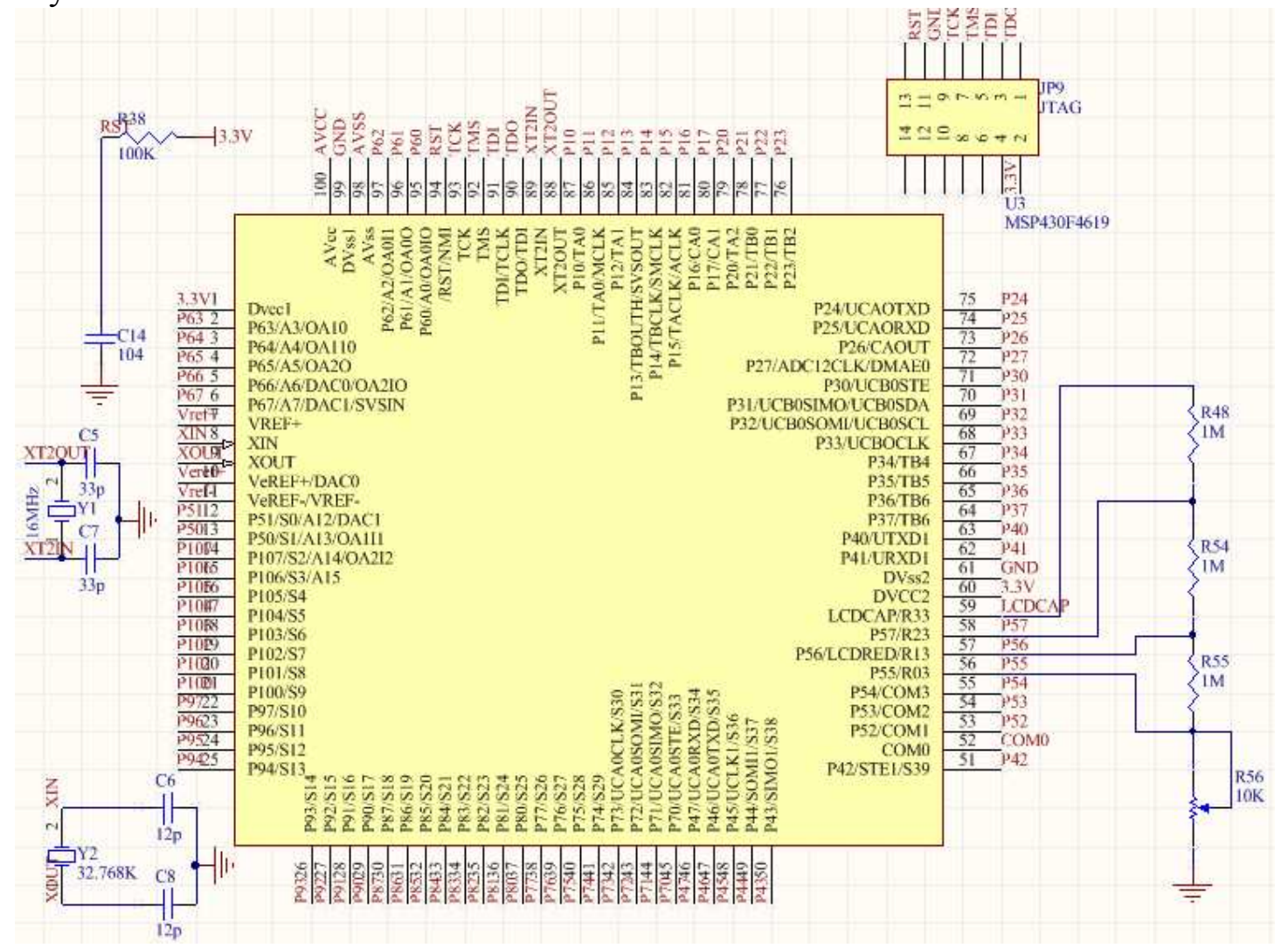

Figure 1 MSP430F4619 minimum system

\section{LCD LCD Module EDS826}

LCD module using EDS826, a total of 6 digits can be displayed, each figure and its corresponding segment code as shown in Figure 2, the LCD module using 4-MUX driver. In the hardware circuit design, connect the EDS826 COM0 to COM3 to MSP430 microcontroller COM0 $\sim \mathrm{COM} 3$, the pin is in turn connected to the MCU S0 to S11. according to the principle of 4-MUX driver, S0 S1 driver and the first tube, S2 and S3 drive second tubes, and so on, S10 and S11 drive sixth tube.

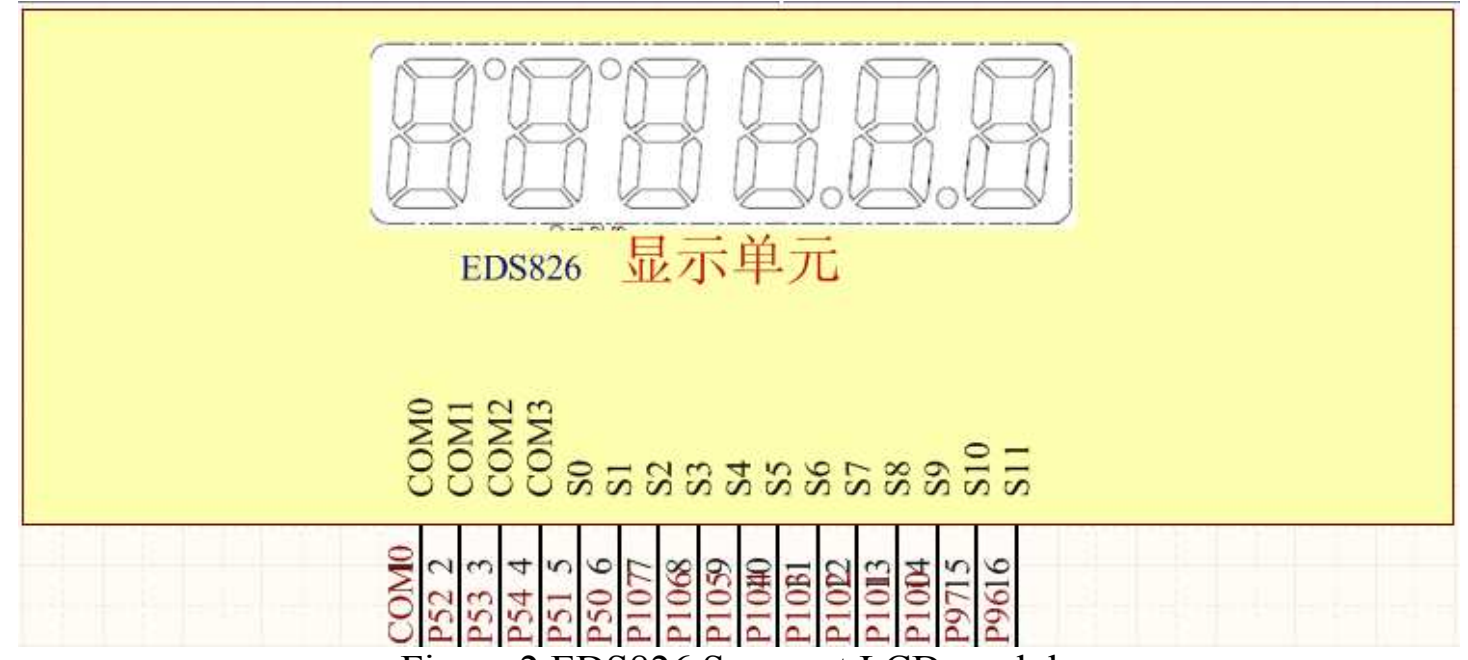

Figure 2 EDS826 Segment LCD module 


\section{LED Lamp Interface Circuit}

16 LED lights connected to the MSP430F4619 microcontroller P7 and P8 port, as shown in Figure 3, through the 3.3V voltage supply, and can be used to control the brightness of the PWM modulation. The brightness of the LED lamp can be controlled according to the brightness of the light with a photosensitive unit. Can also be completed alone water lamp, lamp light off control experiments.
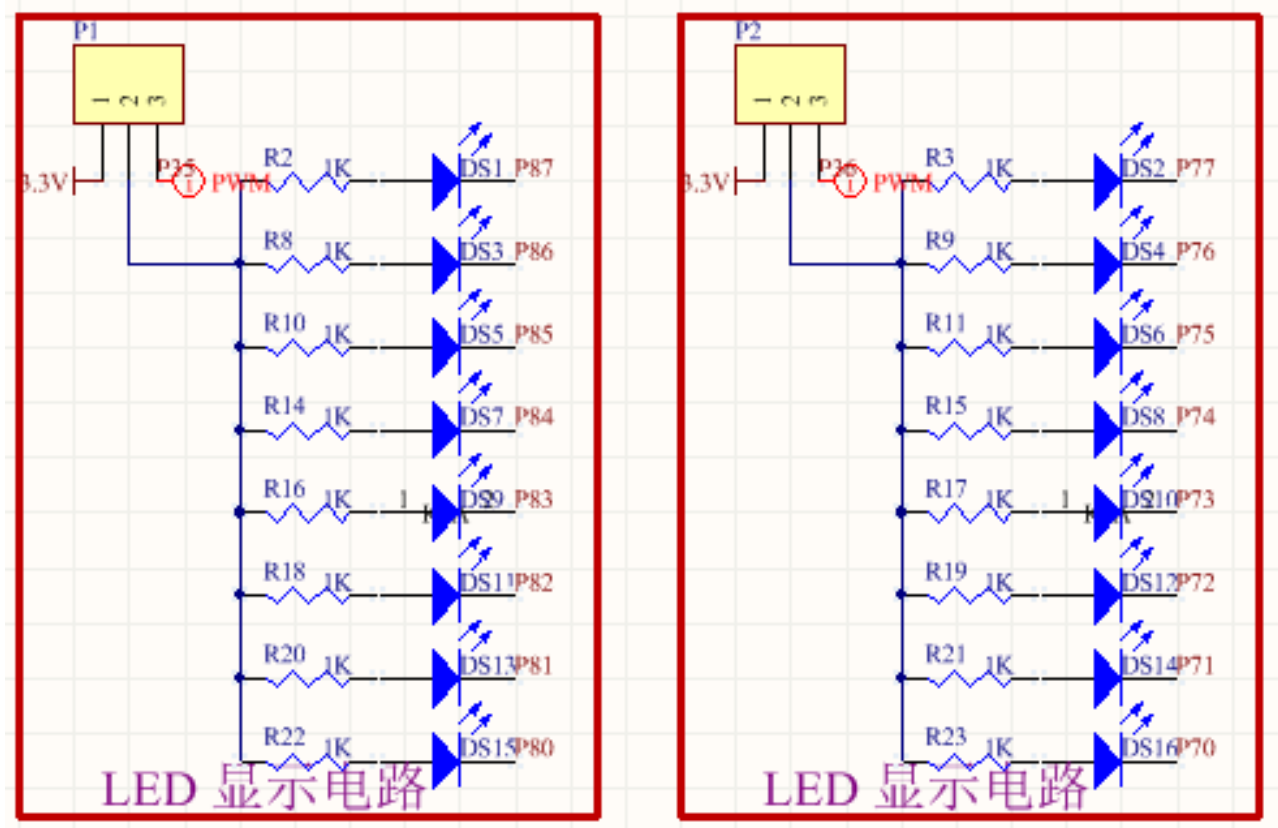

Figure 3 LED display circuit

\section{Test Results}

After the production of printed boards, from the electronic market to buy components and debugging completed, the overall device shown in figure 5. In IAR5.0 on the C language for software programming, write code segment LCD program, the effect shown in figure 6 . Because space is limited.

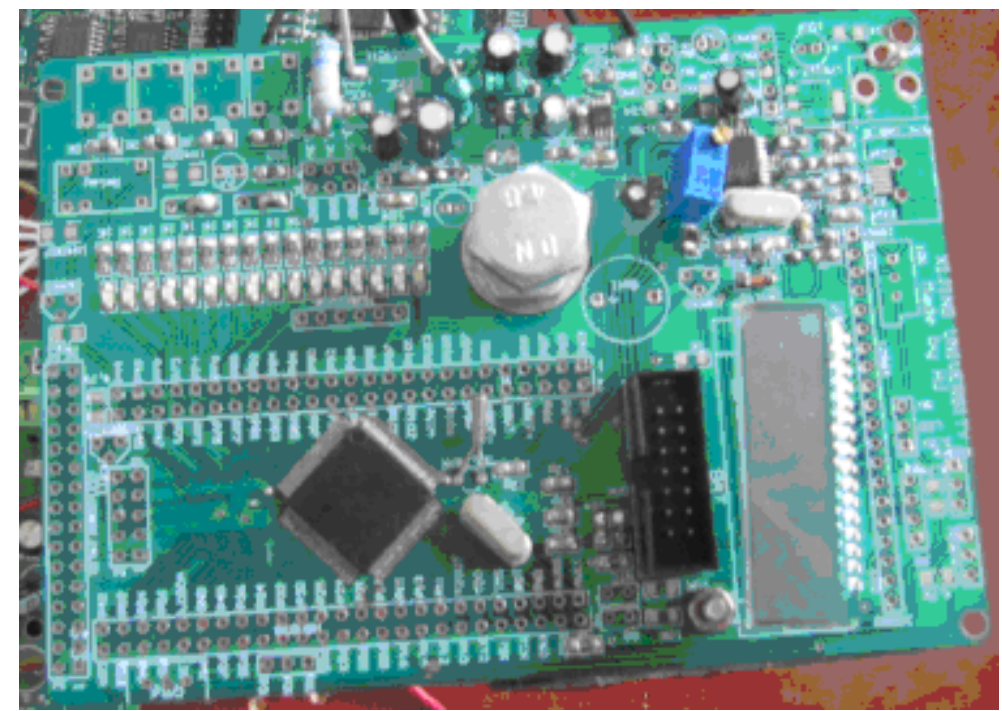

Figure.4 sample of experimental device 


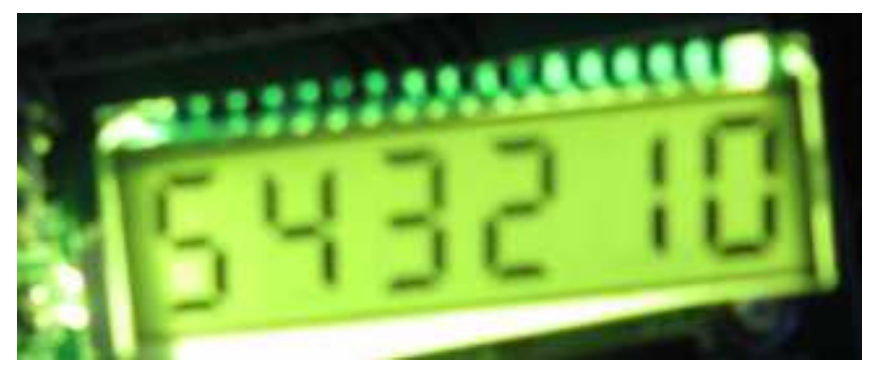

Figure 5 shows the effect

\section{Concluding Remarks}

This paper describes in detail the process of the MSP430F4619 single chip microcomputer experimental device, draws the schematic diagram and makes the PCB printed circuit board. The experimental results show that this kind of MCU interface is rich and powerful, it can realize 8 kinds of experiments, including the basic theory knowledge and practical skills of MCU [14-15]. It is particularly worth mentioning is that the class of $12 \mathrm{~A} / \mathrm{D}$ microcontroller containing a collection of 12 channels, suitable for multi-channel data acquisition, in practical applications can greatly reduce costs. The practice has proved that this kind of device is especially suitable for the teaching practice of computer, electronic information and other professional students, and can be used for the training of College Students' electronic design competition.

\section{Reference:}

[1] Hu Hancai. [M]. single chip microcomputer principle and Interface Technology Third Edition. Beijing: Tsinghua University press, 2010:49-52

[2] Hole Weigong.C51 microcontroller programming and application [M]. Beijing: Electronic Industry Press, 2011:40-56

[3] Zhang Jingwu. The design and Simulation of PROTEUS system based on MCU [M]. Beijing: Publishing House of electronics industry, 2007:21-26

[4] Tangdu instrument company. Experiment instructions [M].2006:21-35

[5] Macro crystal technology.STC89C52 Series MCU manual [M].2011:2-25

[6] Texas Instruments. ultra low power microcontroller [M].2012 MSP430

[7] Xie Kai, Zhao Jian.MSP430 Series MCU system design and engineering practice of [M]. Machinery Industry Press, 2009 